\title{
Optimisation de forme des structures hyperélastiques incompressibles et anisotropes
}

\author{
Abdessalem Jarraya ${ }^{a}$, Imen Kammoun Kallel et Fakhreddine Dammak \\ U2MP, Unité de mécanique, Modélisation et Production, Département de génie mécanique, ENIS, Sfax 3038, Tunisie
}

Reçu le 11 mars 2010, accepté le 5 février 2011

\begin{abstract}
Résumé - Dans ce travail, nous nous sommes intéressés à l'optimisation de forme des structures dont le comportement est hyperélastique incompressible et anisotrope. La fonction énergie est décomposée en une composante isotrope et une composante anisotrope. L'approche décrite dans cet article est le calcul exact de la sensibilité pour les éléments solides en utilisant un matériau à comportement hyperélastique. La résolution du problème mécanique est faite par la méthode des éléments-finis en utilisant un algorithme d'optimisation appelé SQP (Sequential Quadratic Programming). Le critère d'optimisation (fonction objectif à minimiser) est défini à partir du critère de Von Mises avec une limitation de conservation du volume. Les variables d'optimisation sont les coordonnées des points de contrôle avec une paramétrisation par les courbes de B-splines. La faisabilité de la méthode développée est validée par un exemple numérique avec une comparaison du calcul de la sensibilité par la méthode des différences finies.
\end{abstract}

Mots clés : Élément-fini / optimisation de forme / paramétrisation / calcul exact de sensibilité / méthode SQP

\begin{abstract}
Shape optimization of hyperelastic incompressible anisotropic structures. In this paper a shape optimization of hyperelastic incompressible anisotropic structures has been performed. The shape optimization program is implemented by a job control language and a reliable finite-element package program, the SQP (Sequential Quadratic Programming), is used for structural analysis. To achieve the shape optimization, different principles such as structural analysis, sensitivity analysis and mathematical programming are inter-related. The objective is to minimize the Von Mises criterium, with a constraint that the total material volume of the structure remains constant limit for each design variable. In this work, the sensitivity calculation is performed using two methods: numerically by an efficient finite difference scheme and by the exact Jacobian method. The feasibility of the proposed method is carried by a numerical example with anisotropic material.
\end{abstract}

Key words: Finite-elements / shape optimization / parameterization / sensitivity / SQP method

\section{Introduction}

L'optimisation des structures est une des préoccupations essentielles pour la conception des systèmes dans l'industrie mécanique (génie civil, aéronautique, automobile...). Les bureaux d'études ne se contentent pas d'améliorer les performances mécaniques des pièces qu'ils conçoivent, mais ils cherchent également à optimiser leur poids, leur encombrement et leur coût de réalisation.

L'optimisation de forme des structures dont le comportement est non-linéaire a fait l'objet de nombreux travaux depuis deux décennies. Des méthodes performantes

\footnotetext{
a Auteur pour correspondance :

jarraya_abdessalem@yahoo.fr
}

ont été mises au point pour traiter une grande variété de problèmes, nous citons à titre d'exemple :

Barthold et al. [1] ont présenté une comparaison de la performance de deux algorithmes de résolution numérique : la méthode SQP (programmation quadratique séquentielle) et la méthode SAM (méthode d'approximation sphérique). La comparaison a montré que les deux algorithmes SQP et SAM convergent vers la même solution sauf que la deuxième méthode nécessite plus d'itérations. Zingg et al. [2] ont réalisé une comparaison entre un algorithme génétique et un algorithme basé sur le calcul d'un gradient (méthode de l'état adjoint) proposé dans le cadre de différents problèmes d'optimisation de forme aérodynamique. Ils ont démontré que les algorithmes convergent vers la même solution à la différence 
que l'algorithme génétique nécessite plus d'itérations que l'algorithme de type gradient.

Pour obtenir une meilleure conception structurale en changeant la forme de la structure, l'analyse de sensibilité (DSA) est une étape primordiale pour la conception de forme d'une structure. Haug et al. [3] ont prouvé l'existence de la sensibilité pour les systèmes de structures élastiques linéaires. Aucune preuve mathématique n'est disponible sur l'existence ou l'unicité de la sensibilité pour une structure non-linéaire. Dans la prétention de régularité, Santos et al. [4] ont réalisé le calcul de la sensibilité pour des matériaux élastiques non-linéaires. Grindeanu et al. [5] ont développé le calcul de la sensibilité pour des structures à comportement hyperélastique en grande déformation. Le calcul de la sensibilité est réalisé par deux méthodes : la première est la méthode d'état adjoint et la deuxième est la méthode des différences finies telles que les variables d'optimisation sont des variables matérielles. Ces deux méthodes sont comparées aussi par Chunga et al. [6] dans le cadre d'un problème métallurgique. Albert et al. [7] ont présenté deux formulations non-linéaires des approximations multipoints. Ils ont proposé des approximations sur la diagonale de la matrice Hessiénne. Choi et al. [8] ont étudié l'optimisation de forme des structures hyperélastiques. La sensibilité est calculée par la méthode exacte, sauf que les variables d'optimisation sont des variables matérielles de la structure. Dems et al. [9] ont présenté deux approches de l'analyse de la sensibilité pour la variation de forme des structures : la première est la méthode directe et la deuxième est adjointe. Haftka et al. [10] ont réalisé un développement dans l'optimisation des structures et le calcul de la sensibilité. Kim et al. [11] ont étudié l'optimisation de forme des structures hyperélastiques avec contact continu des multiples corps basée sur l'analyse de la sensibilité. Hakim [12] a étudié l'optimisation de forme des structures minces en présence de non linéarité géométrique et matérielle. Il a développé une nouvelle méthode de calcul de sensibilité nommée méthode exacte telle que les variables d'optimisation sont les coordonnées des points de contrôles. Néanmoins cette méthode n'est développée que pour les éléments poutres et coques axisymétriques. Le travail de Merzouki et al. [13] est construit sur la base de principes variationnels pour présenter une comparaison de quatre configurations d'essais hétérogènes en vue d'optimiser l'identification des paramètres de comportement d'un matériau orthotrope élastique.

L'approche décrite dans ce travail est le calcul de la sensibilité par la méthode exacte pour des structures à comportements hyperélastiques incompressibles anisotropes pour les éléments solides. Le contenu de cet article s'appuie sur une comparaison pour le calcul de la sensibilité par la méthode exacte et la méthode des différences finies. Ce manuscrit se compose de quatre sections. Dans la première section on présente les équations constitutives du comportement hyperplasique transversalement isotrope. Dans la deuxième section on présente l'algorithme d'optimisation et la fonction objectif choisie. Le calcul de sensibilité par la méthode exacte fait l'objet de la troisième section et finalement dans la quatrième section on présente les applications numériques et les conclusions.

\section{2 Équations constitutives}

La formulation suivante est une extension du travail développé par Dammak et al. [14] dans le cas des contraintes planes pour un matériau isotrope et transversalement isotrope incompressible. L'objectif est de déterminer le gradient de la fonction objectif et ses limitations en utilisant la méthode exacte avec un matériau à comportement hyperélastique.

Considérons le mouvement de déformation défini par la fonction $\phi(\boldsymbol{X}, t): B \times R \rightarrow R^{3}$ et $\boldsymbol{F}(\boldsymbol{X}, t)$ le gradient de déformation. Ici $\boldsymbol{X} \in R^{3}$ désigne la position d'une particule dans la configuration de référence.

$$
\boldsymbol{F}=\frac{\partial \phi}{\partial \boldsymbol{X}}, J=\operatorname{det}(\boldsymbol{F})
$$

$J$ étant le jacobien de déformation, $\varphi$ est la fonction de transformation qui définit la nouvelle configuration (x) et $B$ est le domaine physique du solide initial. Le tenseur droit de Cauchy-Green, est défini par :

$$
\boldsymbol{C}=\boldsymbol{F}^{\mathrm{T}} \boldsymbol{F}
$$

L'hyperélasticité implique l'existence d'une fonction énergie dépendante du tenseur $\boldsymbol{C}$. Pour le cas d'hyperelasticité isotrope, la fonction énergie est une fonction de $\boldsymbol{C}$ définie par :

$$
W=W\left(I_{1}, I_{2}, I_{3}\right)
$$

où $I_{1}, I_{2}$ et $I_{3}$ sont les trois invariants de $C$ qui sont donnés par :

$$
I_{1}=\operatorname{tr} \boldsymbol{C}, \quad I_{2}=\frac{1}{2}\left[(\operatorname{tr} \boldsymbol{C})^{2}-\left(\operatorname{tr} \boldsymbol{C}^{2}\right)\right], I_{3}=\operatorname{det} \boldsymbol{C}
$$

D'après l'équation (3), la loi d'un matériau hyperélastique isotrope peut être exprimée par les trois premiers invariants de $\boldsymbol{C}$. Pour un matériau hyperélastique transversalement isotrope, d'autres scalaires supplémentaires, ou pseudo-invariants, de $\boldsymbol{C}$ et $\boldsymbol{A}$ sont nécessaires pour décrire l'intégrité de base de ces tenseurs. Les équations suivantes donnent deux invariants scalaires additionnels dont peut dépendre la fonction énergie $W$ dans le cas d'une famille de fibres Holzapfel [15].

$$
I_{4}=\boldsymbol{a}_{0}^{\mathrm{T}} \cdot \boldsymbol{C} \cdot \boldsymbol{a}_{0} \quad I_{5}=\boldsymbol{a}_{0}^{\mathrm{T}} \cdot \boldsymbol{C}^{2} \cdot \boldsymbol{a}_{0} \quad \boldsymbol{A}=\boldsymbol{a}_{0} \otimes \boldsymbol{a}_{0}
$$

avec $\boldsymbol{a}_{0}$ est le champ de vecteur unitaire de la direction d'une famille de fibres.

$I_{4}$ et $I_{5}$ sont des pseudo-invariants qui caractérisent la famille de fibres. En tenant compte de l'incompressibilité du matériau, la fonction énergie ne dépend alors que de quatre invariants avec $I_{3}=1$.

$$
W=W\left(I_{1}, I_{2}, I_{4}, I_{5}\right)
$$


Comme extension de l'hyperélasticité isotrope, nous adoptons la décomposition suivante de la fonction énergie :

$$
W=W_{\text {iso }}\left(I_{1}, I_{2}\right)+W_{\text {aniso }}\left(I_{1}, I_{2}, I_{4}, I_{5}\right)
$$

où $W_{\text {iso }}$ et $W_{\text {aniso sont }}$ respectivement les parties isotrope et anisotrope de la fonction énergie. La fonction énergie libre $W_{\text {iso }}$, responsable du comportement isotrope du matériau peut s'écrire en terme des invariants ou en terme des élongations principales. Dans le cas général, la fonction $W_{\text {aniso }}$ représente la contribution de la famille de fibres et l'interaction entre celles-ci et la matrice. Cette forme de modèle de la fonction énergie libre encadre plusieurs autres modèles pour décrire le comportement transversalement isotrope des élastomères.

Pour la fonction $W_{\text {aniso }}$, plusieurs formes ont été proposées. L'énergie emmagasinée dans les fibres des tissus en biomécanique par exemple, est supposée être une fonction exponentielle Gasser et al. [16].

$$
W_{\text {aniso }}=A_{1}\left(\exp \left[A_{2}\left(I_{4}-1\right)^{2}\right]-1\right)
$$

Cette énergie peut être aussi polynomiale appelée le modèle de renforcement standard défini par :

$$
W_{\text {aniso }}=\frac{1}{2} A\left(I_{4}-1\right)^{2}
$$

Une autre forme de la fonction énergie a été proposée par Bonet et al. [17] pour étendre le modèle transversalement isotrope de St Venant des petites aux grandes déformations.

$$
W_{\text {aniso }}=\left(\alpha+\beta \log (J)+\gamma\left(I_{4}-1\right)\right)\left(I_{4}-1\right)-\frac{1}{2} \alpha\left(I_{5}-1\right)
$$

D'après la décomposition de l'équation (7), le tenseur des contraintes de Piola-Kirchhoff se décompose en deux parties sous la forme suivante:

$$
\boldsymbol{S}=2 \frac{\partial W}{\partial \boldsymbol{C}}=\boldsymbol{S}_{\text {iso }}+\boldsymbol{S}_{\text {aniso }}
$$

$\boldsymbol{S}_{\text {iso }}$ est le tenseur de contrainte Piola-Kirchhoff isotrope et $\boldsymbol{S}_{\text {aniso }}$ est le tenseur de contrainte Piola-Kirchhoff anisotrope.

Le module tangent se décompose également en deux parties :

$$
\mathbb{C}=2 \frac{\partial \boldsymbol{S}}{\partial \boldsymbol{C}}=\mathbb{C}_{\text {iso }}+\mathbb{C}_{\text {aniso }}
$$

$\mathbb{C}_{\text {iso }}$ est le module tangent isotrope et $\mathbb{C}_{\text {aniso }}$ est le module tangent anisotrope.

\section{Fonction objectif}

Un problème général d'optimisation peut être formulé de la manière suivante :

$$
\begin{aligned}
& \operatorname{Minf}(\boldsymbol{v}) \\
& \boldsymbol{v} \in \mathbb{R}^{n}
\end{aligned}
$$

avec les limitations

$$
\begin{array}{ll}
h_{j}(\boldsymbol{v})=0 & j=1, \ldots \ldots \ldots \ldots, m \\
g_{k}(\boldsymbol{v}) \leq 0 & k=1, \ldots \ldots \ldots, p \\
v_{i l} \leq v_{i} \leq v_{i u} & i=1,2, \ldots \ldots \ldots, n
\end{array}
$$

où $f\left(\boldsymbol{v}_{i}\right)$ est la fonction objectif, $\boldsymbol{v}_{i}$ est le vecteur des $n$ variables indépendantes, $h_{j}(\boldsymbol{v})$ sont les $m$ limitations d'égalité et $g_{k}(\boldsymbol{v})$ sont les $p$ limitations d'inégalité. $v_{i l}$ et $v_{i u}$ sont respectivement les deux limitations géométriques inférieure et supérieure pour la variable d'optimisation $v_{i}$.

Pour résoudre ce type de problème on a choisi la méthode SQP car elle nous permet de modéliser les équations (13) et (14) en un point donné $v_{i}$ par un sous-problème de programmation quadratique, et d'utiliser la solution de ce sous-problème pour construire une meilleure approximation $v_{i+1}$. Ce processus est répété en produisant une succession d'approximations, qui doivent converger vers la solution $v^{*}$. La méthode SQP possède quelques propriétés qui font leur intérêt pratique, la première est qu'elle ne nécessite pas une solution initiale qui satisfait toutes les limitations de l'équation (14). La seconde est que son succès dans la résolution dépend de l'existence et de la validité des algorithmes de résolution des problèmes quadratiques.

L'objectif dans notre étude est de réduire la contrainte maximale dans la structure, avec certaines limitations géométriques satisfaisantes. En tenant compte de tous ces soucis, nous avons élaboré un critère d'optimisation qui est une fonction continue et différentiable. La fonction objectif est la contrainte équivalente de Von Mises.

$$
f(v, \boldsymbol{U})=\operatorname{Min} \frac{1}{2} \int_{V} S_{\text {eq }}^{2} \mathrm{~d} V
$$

où $S$ est le tenseur de contrainte de Piola-Kirchhoff, $S_{\text {eq }}$ est la contrainte équivalente de Von Mises en déformations planes définie par :

$$
\begin{aligned}
& S_{\text {eq }}= \\
& \sqrt{0.5\left[\left(S_{11}-S_{22}\right)^{2}+\left(S_{22}-S_{33}\right)^{2}+\left(S_{33}-S_{11}\right)^{2}+6 S_{12}^{2}\right]}
\end{aligned}
$$

avec une limitation de conservation de volume le long du processus d'optimisation

$$
g=\int_{V} \mathrm{~d} V-V_{0}=0
$$

La fonction objectif du phénomène d'optimisation de la structure est la somme algébrique de toutes les fonctions objectifs élémentaires

$$
\begin{aligned}
f & =\sum_{e=1}^{n e l t} f_{e}=\sum_{e=1}^{n e l t} \frac{1}{2} \int_{V_{e}} S_{\text {eq }}^{2} \mathrm{~d} V=\sum_{e=1}^{n e l t} \frac{1}{2} \int_{V_{\xi}} S_{\text {eq }}^{2} \operatorname{det} \boldsymbol{J} \mathrm{d} V_{\xi} \\
\text { et } g & =\sum_{e=1}^{\text {nelt }} g_{e}=\sum_{e=1}^{\text {nelt }} \int_{V_{e}} \mathrm{~d} V_{e}=\sum_{e=1}^{\text {nelt }} \int_{V_{\xi}} \operatorname{det} \boldsymbol{J} \mathrm{d} V_{\xi}
\end{aligned}
$$


telle que $f_{e}$ et $g_{e}$ sont respectivement la fonction objectif et la fonction limitation pour un élément-fini et nelt est le nombre d'éléments-finis de la structure discrétisée.

\section{Analyse de sensibilité}

Nous présentons dans cette section la méthode analytique discrète pour le calcul des sensibilités de la fonction objectif f $f=f\left(v_{i}, \boldsymbol{U}\right)$ qui sont fortement non-linéaires selon les $n_{e q}$ variables d'état (déplacement), elles-mêmes selon les $n$ variables d'optimisation, où $v_{i}$ est le vecteur des coordonnées de points de contrôles et $\boldsymbol{U}$ est le vecteur déplacement.

Le problème d'analyse de la sensibilité consiste à la dérivation de la fonction objectif discrète et les limitations par rapport aux variables d'optimisation tout en satisfaisant les équations non-linéaires d'équilibre.

$$
\boldsymbol{R}(\boldsymbol{v}, \boldsymbol{U})=\boldsymbol{F}_{\text {int }}-\boldsymbol{F}_{\text {ext }}=0
$$

où $\boldsymbol{R}(\boldsymbol{v}, \boldsymbol{U})$ est le vecteur résidu global, $\boldsymbol{F}_{\text {int }}$ et $\boldsymbol{F}_{\text {ext }}$ sont respectivement les travaux des efforts internes et externes.

Le problème d'analyse de sensibilités consiste à calculer la dérivée totale de $f$ en $(\boldsymbol{v}, \boldsymbol{U})$ tel que :

$$
\frac{\mathrm{d} f}{\mathrm{~d} v_{i}}=\frac{\partial f}{\partial v_{i}}+\left(\frac{\partial f}{\partial \boldsymbol{U}}\right)^{T}\left(\frac{\partial \boldsymbol{U}}{\partial v_{i}}\right) i=1, n
$$

L'effort intérieur est défini par :

$$
\boldsymbol{F}_{\text {int }}=\sum_{e=1}^{\text {nelt }} \boldsymbol{F}_{\text {int }}^{e}=\sum_{e=1}^{\text {nelt }} \int_{V_{e}} \boldsymbol{B}^{\mathrm{T}} \boldsymbol{S} \mathrm{d} V_{e}=\sum_{e=1}^{\text {nelt }} \int_{V_{\xi}} \boldsymbol{B}^{T} \boldsymbol{S} \operatorname{det} \boldsymbol{J} \mathrm{d} V_{\xi}
$$

avec $\boldsymbol{B}$ la matrice reliant les déformations aux déplacements.

Dans notre étude, l'effort extérieur est donné par la relation suivante :

$$
\boldsymbol{F}_{\text {ext }}=\int_{\xi} \boldsymbol{N} P J_{\xi} \mathrm{d} \xi
$$

telle que $\boldsymbol{N}$ est la matrice des fonctions d'interpolations, $P$ est la pression appliquée, $J_{\xi}$ est la courbe d'intégration et $\xi$ est un paramètre.

La dérivée du résidu global par rapport aux coordonnées des points de contrôles est donnée par :

$$
\frac{\partial \boldsymbol{R}}{\partial \boldsymbol{v}_{i}}=\frac{\partial \boldsymbol{F}_{\text {int }}}{\partial v_{i}}-\frac{\partial \boldsymbol{F}_{\text {ext }}}{\partial v_{i}}
$$

La variation de l'effort intérieur élémentaire par rapport aux cordonnées des points de contrôles est exprimée par :

$$
\begin{aligned}
\frac{\partial \boldsymbol{F}_{\text {int }}^{e}}{\partial v_{i}}=\int_{V_{\xi}}\left[\boldsymbol { B } ^ { \mathrm { T } } \left(\frac{\partial \boldsymbol{S}}{\partial v_{i}}\right.\right. & \left.\operatorname{det} \boldsymbol{J}+\boldsymbol{S} \frac{\partial \operatorname{det} \boldsymbol{J}}{\partial v_{i}}\right) \\
& \left.+\left(\frac{\partial \boldsymbol{B}}{\partial v_{i}}\right)^{\mathrm{T}} \boldsymbol{S} \operatorname{det} \boldsymbol{J}\right] \mathrm{d} V_{\xi}
\end{aligned}
$$

La variation de l'effort extérieur par rapport aux variables d'optimisation est exprimée par :

$$
\frac{\partial \boldsymbol{F}_{\text {ext }}}{\partial v_{i}}=\int_{\xi} P\left(\frac{\partial \boldsymbol{N}}{\partial v_{i}} \boldsymbol{J}_{\xi}+\boldsymbol{N} \frac{\partial \boldsymbol{J}_{\xi}}{\partial v_{i}}\right) \mathrm{d} \xi
$$

Finalement le gradient du résidu global est défini par :

$$
\frac{\mathrm{d} \boldsymbol{R}}{\mathrm{d} v_{i}}=\frac{\partial \boldsymbol{R}}{\partial v_{i}}+\frac{\partial \boldsymbol{R}}{\partial \boldsymbol{U}} \frac{\partial \boldsymbol{U}}{\partial v_{i}}=0
$$

tel que :

$$
\frac{\partial \boldsymbol{R}}{\partial \boldsymbol{U}}=\boldsymbol{K}_{\mathrm{T}}
$$

où $\boldsymbol{K}_{\mathrm{T}}$ est la matrice tangente globale à l'équilibre.

À partir de l'équation (23)-(26) on a la relation suivante :

$$
\boldsymbol{K}_{\mathrm{T}} \frac{\partial U}{\partial v_{i}}=-\frac{\partial \boldsymbol{R}}{\partial v_{i}}=-\left(\frac{\partial \boldsymbol{F}_{\text {int }}}{\partial v_{i}}-\frac{\partial \boldsymbol{F}_{\mathrm{ext}}}{\partial v_{i}}\right)
$$

La résolution de l'équation (28) permet d'avoir les termes $\frac{\partial \boldsymbol{U}}{\partial v_{i}}$ qui peuvent être calculés par la méthode des différences finies ou la méthode exacte.

Les détails de ce calcul ont été développés par Abid et al. [18].

\subsection{Sensibilité du volume}

L'expression du volume pour une structure modélisée par éléments-finis est donnée par :

$$
V=\int_{V} \mathrm{~d} V=\sum_{e=1}^{n e l t} \int_{V_{e}} \mathrm{~d} V_{e}
$$

où $\mathrm{d} V_{e}=\operatorname{det} \boldsymbol{J} \mathrm{d} V_{\xi}$ est le volume élément-fini, $\boldsymbol{J}$ est la matrice jacobienne et $\mathrm{d} V_{\xi}$ est le volume élémentaire de référence.

Le gradient du volume élémentaire par rapport aux variables d'optimisation est :

$$
\frac{\partial V_{e}}{\partial v_{i}}=\int_{V_{\xi}} \frac{\partial \operatorname{det} \boldsymbol{J}}{\partial v_{i}} \mathrm{~d} V_{\xi}
$$

Le gradient de chaque terme de la matrice jacobienne sera défini par un calcul de la dérivée des fonctions d'interpolations selon chaque direction.

\subsection{Sensibilité de la contrainte}

On suppose que $f$ et $\boldsymbol{U}$ sont deux fonctions continues et dérivables, telles que le gradient de la fonction objectif élémentaire est donné par l'équation :

$$
\frac{\partial f_{e}}{\partial v_{i}}=\int_{V_{\xi}}\left[S_{\mathrm{eq}} \frac{\partial S_{\mathrm{eq}}}{\partial v_{i}} \operatorname{det} \boldsymbol{J}+\frac{1}{2} S_{\mathrm{eq}}^{2} \frac{\partial \operatorname{det} \boldsymbol{J}}{\partial v_{i}}\right] \mathrm{d} V_{\xi} i=1, n
$$




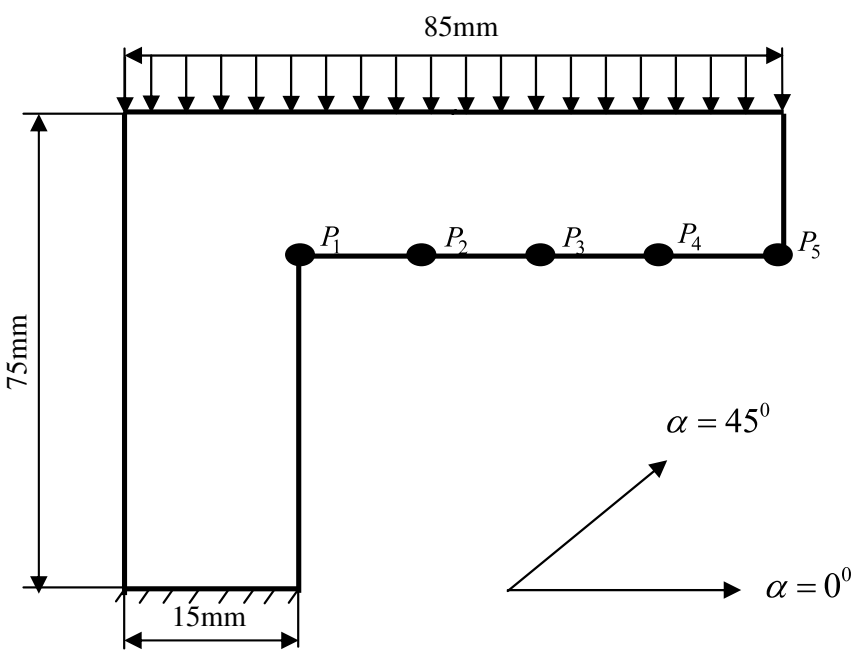

$P_{5}(85,55)$

Fig. 1. Géométrie initiale de la structure avec les points de contrôles.

La sensibilité peut être évaluée en utilisant le deuxième tenseur de contrainte de Piola-Kirchoff $\boldsymbol{S}$ qui est donné par l'expression (11).

D'où le gradient des termes du tenseur $\boldsymbol{S}$ par rapport aux variables d'optimisation est donné par l'expression suivante :

$$
\frac{\partial \boldsymbol{S}_{\text {iso }}}{\partial v_{i}}=\frac{1}{2} \mathbb{C}_{\text {iso }}: \frac{\partial \boldsymbol{C}}{\partial v_{i}} \quad \text { et } \quad \frac{\partial \boldsymbol{S}_{\text {aniso }}}{\partial v_{i}}=\frac{1}{2} \mathbb{C}_{\text {aniso }}: \frac{\partial \boldsymbol{C}}{\partial v_{i}}
$$

où $\mathbb{C}_{\text {iso }}$ et $\mathbb{C}_{\text {aniso }}$ sont respectivement le module tangent isotrope et le module anisotrope définis par :

$$
\mathbb{C}_{\text {iso }}=4 \frac{\partial^{2} W_{\text {iso }}}{\partial \boldsymbol{C} \partial \boldsymbol{C}} \quad \text { et } \quad \mathbb{C}_{\text {aniso }}=4 \frac{\partial^{2} W_{\text {aniso }}}{\partial \boldsymbol{C} \partial \boldsymbol{C}}
$$

La variation de la contrainte équivalente de Von Mises par rapport aux variables d'optimisation est donnée par :

$$
\begin{aligned}
& \frac{\partial S_{\mathrm{eq}}}{\partial v_{i}}=\frac{1}{2 S_{\mathrm{eq}}} \\
& \times\left[\begin{array}{l}
\left(S_{11}-S_{22}\right)\left(\frac{\partial S_{11}}{\partial v_{i}}-\frac{\partial S_{22}}{\partial v_{i}}\right)+\left(S_{22}-S_{33}\right) \\
\times\left(\frac{\partial S_{22}}{\partial v_{i}}-\frac{\partial S_{33}}{\partial v_{i}}\right) \\
+\left(S_{33}-S_{11}\right)\left(\frac{\partial S_{33}}{\partial v_{i}}-\frac{\partial S_{22}}{\partial v_{i}}\right)+3\left(S_{12} \frac{\partial S_{12}}{\partial v_{i}}\right)
\end{array}\right]
\end{aligned}
$$

On calcule de la même manière le gradient $\frac{\partial f_{e}}{\partial u_{k}}$ $(k=1$, ndim $\times$ nnode $)$.

\section{Résultats numériques}

Dans le but de montrer la performance du développement réalisé avec un matériau à comportement hyperélastique incompressible anisotrope, nous étudions un

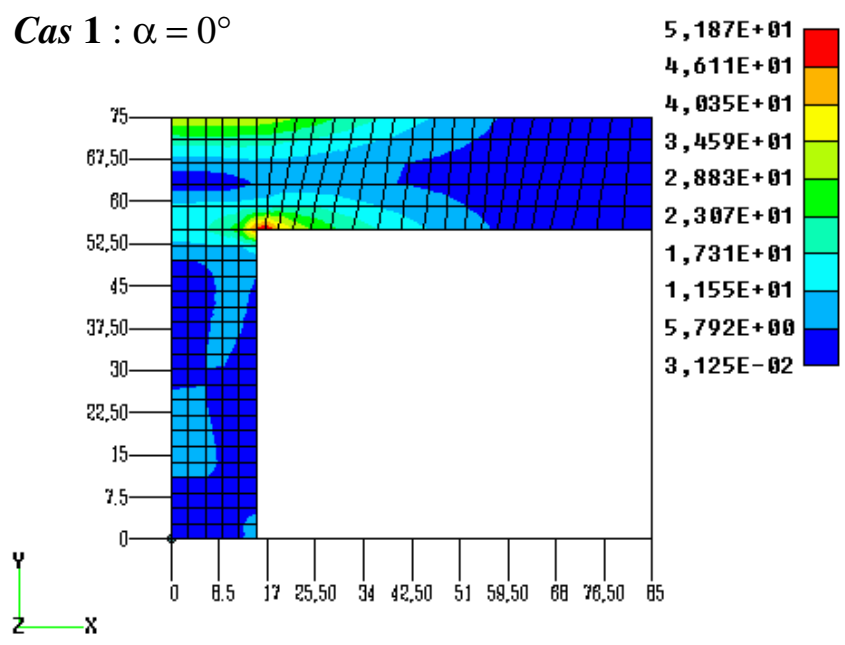

Fig. 2. Distribution de la contrainte de Von Mises avant optimisation.

exemple pour deux cas d'orientation des fibres $\alpha=0^{\circ}$ et $\alpha=45^{\circ}$. La discrétisation de la structure est réalisée par l'élément fini quadrilatéral. Nous avons utilisé la fonction énergie qui s'écrit sous la forme suivante :

$$
W_{\text {iso }}=C_{10}\left(I_{1}-3\right) \quad \text { avec } \quad C_{10}=100 \mathrm{MPa}
$$

La partie anisotrope est donnée par :

$$
W_{\text {aniso }}=\frac{A_{1}}{2}\left(I_{4}-1\right)^{2}+A_{2}\left(I_{1}-3\right)\left(I_{4}-1\right)
$$

avec $A_{1}=200 \mathrm{MPa}$ et $A_{2}=1 \mathrm{MPa}$.

\subsection{Portique en forme de $L$}

La géométrie de la structure étudiée est représentée sur la figure 1. Nous avons utilisé cinq points de contrôle qui définissent la forme optimisée de la structure. La 

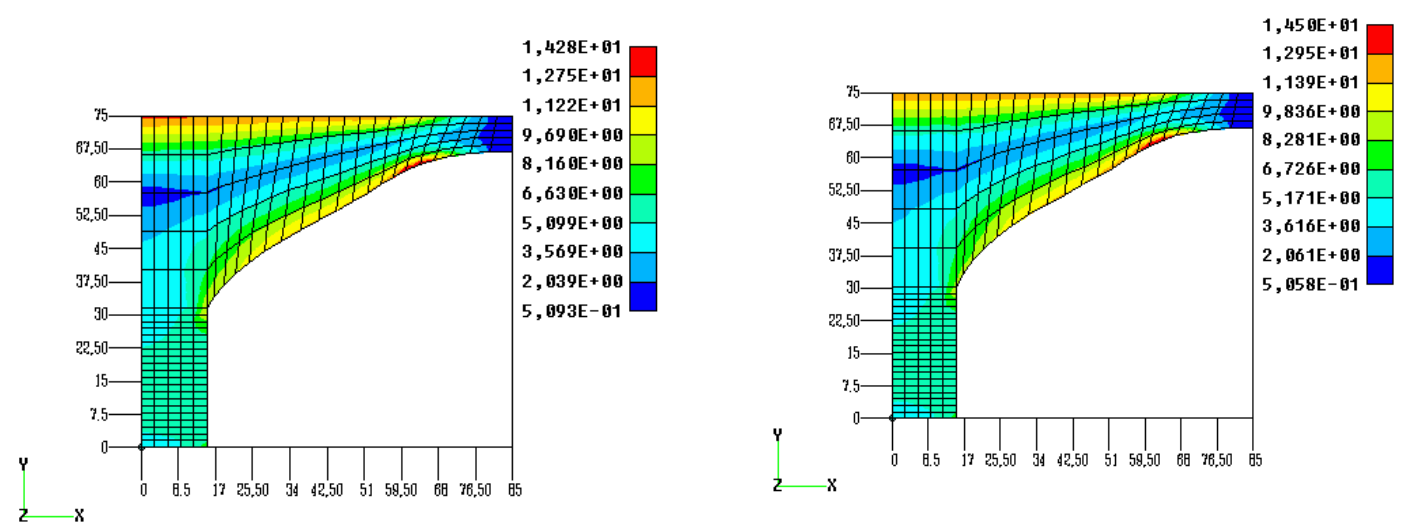

Fig. 3. Distribution de la contrainte de Von Mises après optimisation en MPa. (a) Exacte. (b) DF.

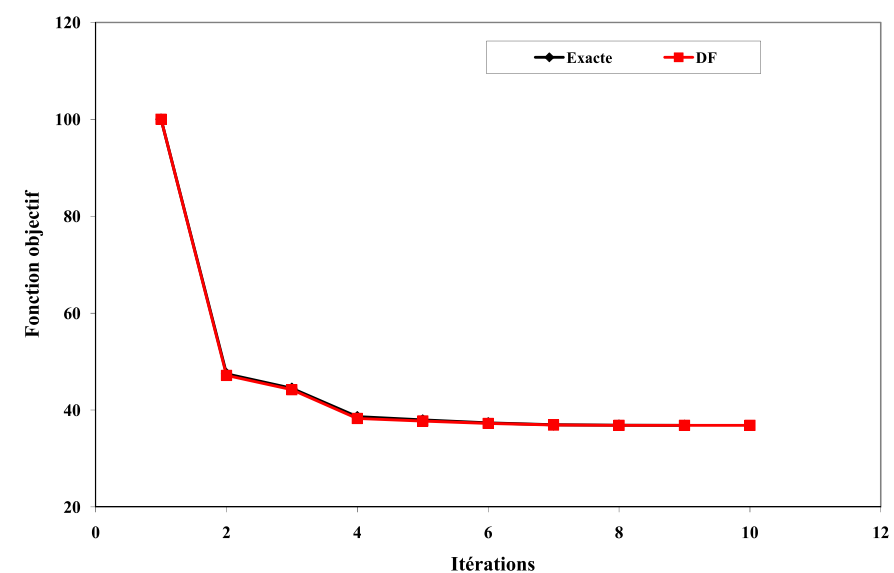

Fig. 4. Évolution de la fonction objectif au cours des itérations.

structure est discrétisée par 225 éléments-finis quadrilatéraux. La structure est soumise à un chargement de $1 \mathrm{~N} . \mathrm{mm}^{-2}$. La figure 2 représente la distribution de la contrainte de Von Mises avant optimisation avec une orientation des fibres de $\alpha=0^{\circ}$. La distribution de la contrainte moyenne de Von Mises après optimisation avec la méthode exacte et la méthode différences finies est représentée sur la figure 3. Enfin, sur la figure 4 est représentée l'évolution de la fonction objectif au cours des itérations. Dans le cas où l'orientation des fibres est de $\alpha=45^{\circ}$ la distribution de la contrainte moyenne de Von Mises avant optimisation et après optimisation avec les deux méthodes de calcul de sensibilité est représenté respectivement sur les figures 5 et 6 . L'évolution de la fonction objectif aux cours des itérations est représentée sur la figure 7. Enfin le tableau 1 représente une comparaison des résultats.

On peut conclure à partir de ce tableau qu'on a une réduction de $77 \%$ de la contrainte moyenne de Von Mises après optimisation avec une conservation du volume total de la structure. On remarque aussi que le calcul de la sensibilité par la méthode exacte a permis un gain du temps de calcul considérable par rapport à l'utilisation de la méthode des différences finies.

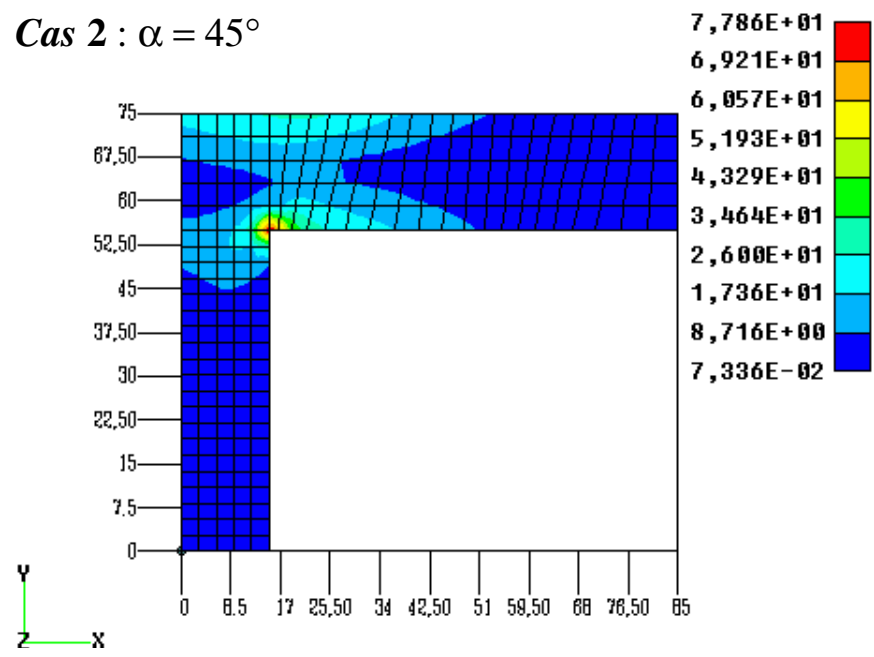

Fig. 5. Distribution de la contrainte de Von Mises avant optimisation en MPa.

\section{Conclusions}

Dans ce travail, nous avons présenté l'optimisation de forme des structures hyperélastiques incompressibles anisotropes qui subissent de grandes déformations avec des non-linéarités géométriques et matérielles. Dans cette approche, les paramètres d'optimisations, sont calculés en résolvant un problème d'optimisation non-linéaire.

La structure a été discrétisée par des élémentsfinis quadrilatéraux. La résolution du problème nonlinéaire est effectuée avec la programmation quadratique séquentielle SQP. Le développement d'une nouvelle méthode de calcul de sensibilité par rapport aux coordonnées des points de contrôles est réalisé pour des éléments solides avec un comportement hyperélastique anisotrope. Nous pouvons conclure qu'en utilisant la méthode exacte nous avons une réduction importante du temps de calcul par rapport à la méthode de différences finies. 


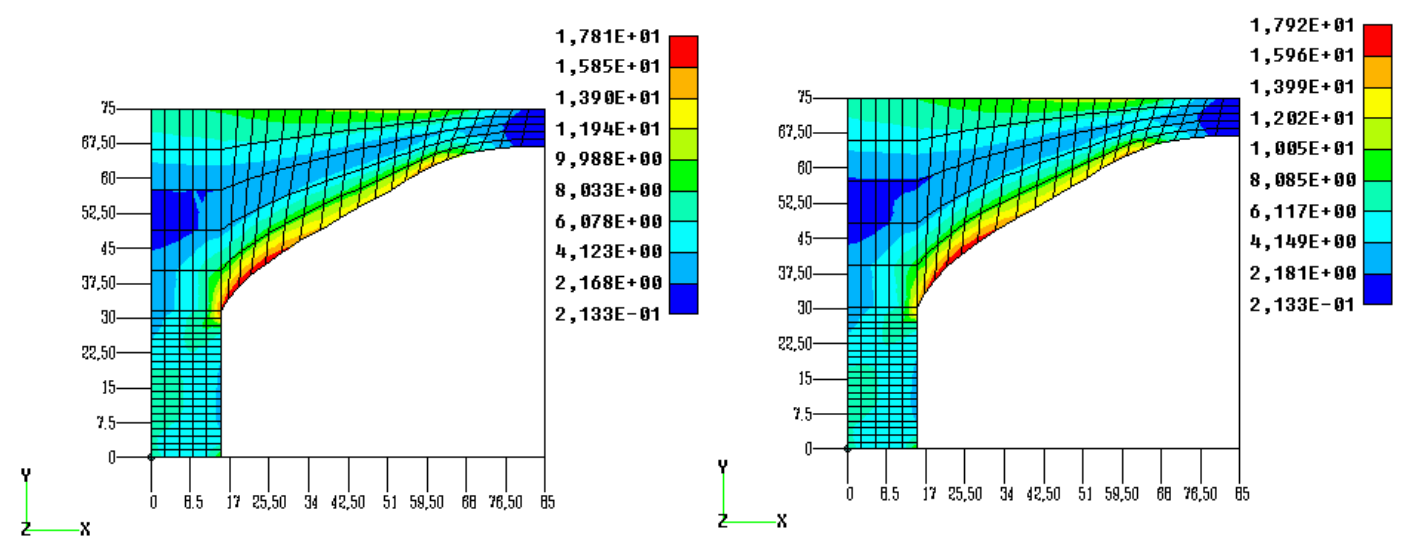

Fig. 6. Distribution de la contrainte de Von Mises après optimisation en MPa. (a) Exacte. (b) DF.

Tableau 1. Comparaison des résultats.

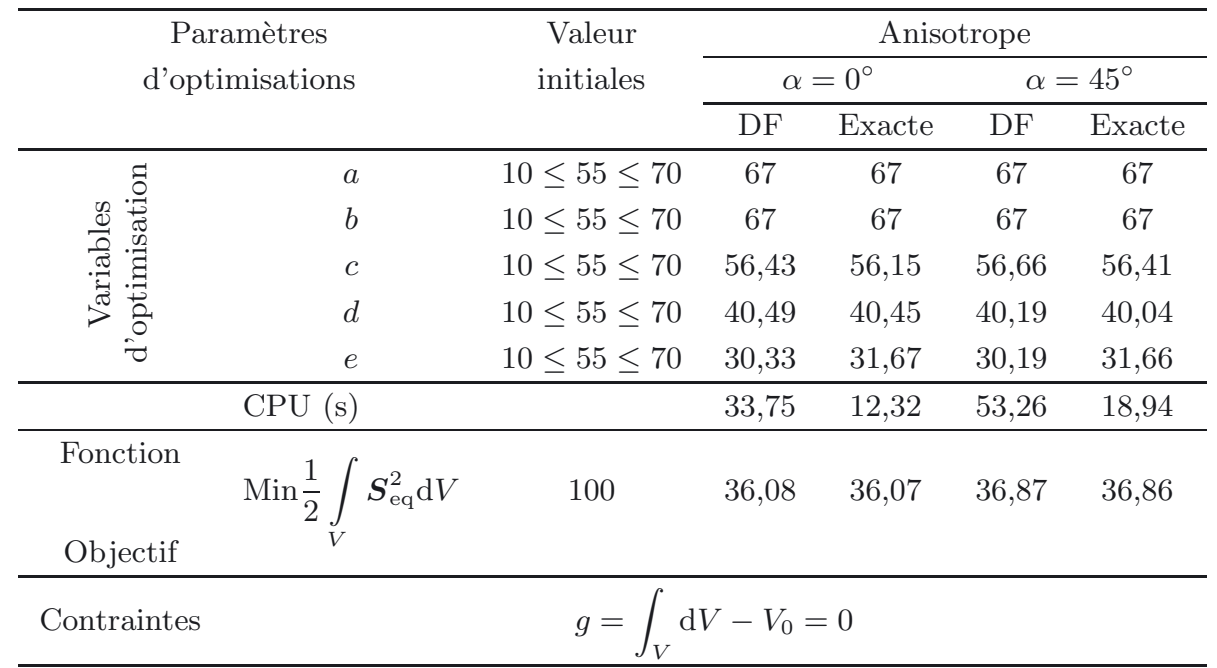

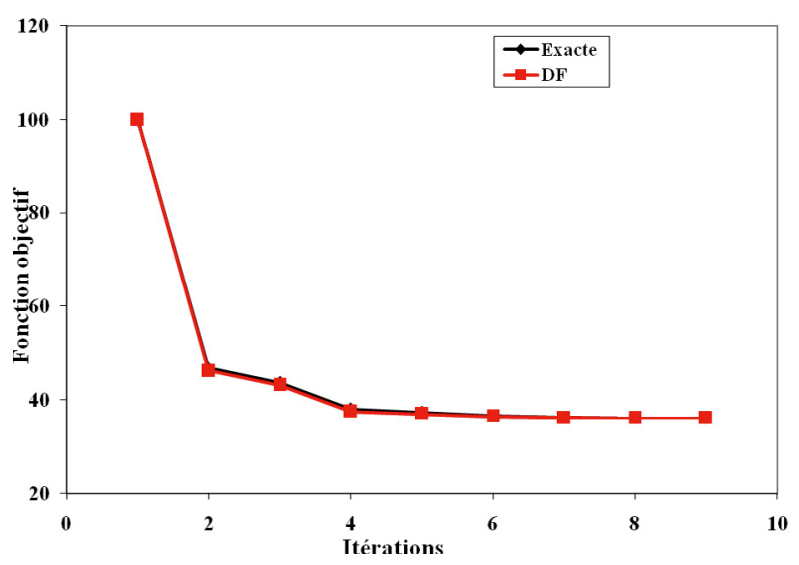

Fig. 7. Évolution de la fonction objectif au cours des itérations.

\section{Références}

[1] F.-J. Barthold, N. Stander, E. Stein, Performance comparaison of SAM and SQP methods for structural shape optimization, Struct. Multidisc. Optim. 11 (1996) 102112
[2] D. Zingg, M. Nemec, T. Pulliam, A comparative evaluation of genetic and gradient-based algorithms applied to aerodynamic optimization, Revue Européenne de Mécanique Numérique 17 (2008) 103-126

[3] E.J. Haug, K.K. Choi, V. Komkov, Design sensitivity analysis of structural systems, London, Academic Press, 1986

[4] J.L.T. Santos, K.K. Choi, Shape design sensitivity analysis of nonlinear structural systems, Struct. Optim. 4 (1992) 23-35

[5] I. Grindeanu, K.H. Chang, K.K. Choi, J.S. Chen, Design sensitivity analysis of hyper-elastic structures using a meshless method, AIAA J. 37 (1999) 990-997

[6] S.H. Chunga, Y.S. Kwonb, S.J. Parkc, R.M. Germand, Sensitivity analysis by the adjoint variable method for optimization of the die compaction process in particulate materials processing, Finite Elem. Anal. Des. 45 (2009) 836-844

[7] A. Albert, L.F. Groenwold, P. Etman, Sequential approximate optimization using dual subproblems based on incomplete series expansions, Struct. Multidisc. Optim. 36 (2008) $547-570$

[8] K.K. Choi, W. Duan, Design sensitivity analysis and shape optimization of structural components with hyperelastic material, Comput. Meth. Appl. Mech. Eng. 187 (2000) 219-243 
[9] K. Dems, R.T. Haftka, Two approaches to sensitivity analysis for shape variation of structures, Mech. Struct. 16 (1988) 501-522

[10] R.T. Haftka, H.L.M. Adelman, Recent developments in structural sensitivity analysis, Struct. Opt. 1 (1989) 137151

[11] N.H. Kim, Optimization of a hyper-elastic structure with multi body contact using continuum-based shape design sensitivity analysis, Struct. Opt. 21 (2001) 196-208

[12] N. Hakim, Contribution à l'optimisation de forme de structures minces en présence de non linéarités géométriques et matérielles, Thèse, Université de Technologie de Compiègne, 1998

[13] T. Merzouki, F. Meraghni, H. Chalal, T. Ben Zineb, Comparaison de configurations d'essais hétérogènes en vue d'améliorer l'identification du comportement élastique orthotrope par l'erreur en relation de comportement, Mécanique \& Industries 10 (2009) 351-364
[14] F. Dammak, A. Regaieg, I.K. Kammoun, A. Dhiab, Modélisation de la loi de comportement hyperélastique transversalement isotrope des élastomères, Revue Européenne de Mécanique Numérique 16 (2007) 103-126

[15] G.A. Holzapfel, Non linear solid mechanics, Wiley, 2000

[16] G.A. Gasser, G.A. Holzapfel, A rate-independent elastoplastic constitutive model for fiber-reinforced composites at finite strains: continuum basis, algorithmic formulation and finite element implementation, Comput. Mech. 29 (2002) 340-360

[17] J. Bonet, A.J. Burton, A simple orthotropic, transversely isotropic hyperelastic constitutive equation for large strain computations, Comput. Meth. Appl. Mech. Eng. 162 (1998) 151-164

[18] S. Abid, A. Jarraya, F. Dammak, M. Haddar, Design sensitivity and shape optimization of geometrical non linear structure, Mécanique \& Industries 9 (2008) 17-23 